\title{
Holographic Bound in Quantum Field Energy Density and Cosmological Constant
}

\author{
Paolo Castorina \\ Dipartimento di Fisica, Università di Catania, and INFN Sezione di Catania, Catania, Italia \\ Email: paolo.castorina@ct.infn.it
}

Received January 22, 2013; revised March 8, 2013; accepted April 10, 2013

Copyright (C) 2013 Paolo Castorina. This is an open access article distributed under the Creative Commons Attribution License, which permits unrestricted use, distribution, and reproduction in any medium, provided the original work is properly cited.

\begin{abstract}
The cosmological constant problem is reanalyzed by imposing the limitation of the number of degrees of freedom (d.o.f.) due to entropy bounds directly in the calculation of the energy density of a field theory. It is shown that if a quantum field theory has to be consistent with gravity and holography, i.e. with an upper limit of storing information in a given area, the ultraviolet momentum cut-off is not the Planck mass, $M_{p}$, as naively expected, but $M_{p} / N_{U}^{1 / 4}$ where $N_{U}$ is the number of d.o.f. of the universe. The energy density evaluation turns out completely consistent with Bousso's bound on the cosmological constant value. The scale $M_{p} / N_{U}^{1 / 4}$, that in the "fat graviton" theory corresponds to the graviton size, originates by a self-similar rearrangement of the elementary d.o.f. at different scales that can be seen as an infrared-ultraviolet connection.
\end{abstract}

Keywords: Cosmological Constant; Holography; Quantum Fields

\section{Introduction}

In 1955, von Weizsaker [1] started a program to derive quantum theory by postulating a fundamental quantized binary alternative, called "ur", and, many years later, Wheeler [2] suggested that information theory must play a relevant role in understanding the foundations of quantum mechanics, the "It for bit" proposal.

On the other hand, it is now generally accepted that the picture of space-time as a locally flat Minkowsky manifold breaks down at distances of order of Planck scale, $l_{p} \simeq 1.6 \times 10^{-33} \mathrm{~cm}$, and that, due to space-time uncertainty $[3,4]$, an elementary area $A_{p}=l_{p}^{2}$ should be considered as a fundamental space-time cell.

The previous ideas are the starting points of the main approaches to quantum gravity that consider quantum space-time to be discrete: loop quantum gravity $[5,6]$ and string theory $[7,8]$.

Moreover, by combining the assumptions of a quantized fundamental (binary) degree of freedom (d.o.f.) and of an elementary area, $A_{p}$, with Bekenstein's result on black-hole entropy [9], the Holographic Principle emerges [10-18]:

a) the number of possible states in a region of space is the same as that of a system of binary d.o.f. distributed on the causal horizon of the region;

b) the number of d.o.f., $N$, of a region of space is bounded by the area, $A$, in unit of the elementary area $A_{p} \quad(c=\hbar=1)$ :

$$
N \leq \frac{A}{A_{p}}
$$

Holography has a crucial role in the description of gravity as an emergent phenomenon of thermodynamical origin [19-22] and/or as an entropic force [23].

Moreover, following Banks's proposal [18] that the cosmological constant, $\Lambda$, should be related to the number of d.o.f. in the fundamental theory, it has been shown [12] that in any universe with a positive cosmological constant one obtains, by holographic entropy bounds, $[10,12]$ an upper limit to the number of d.o.f. of the universe, $N_{U}$, given by

$$
N_{U} \leq \frac{3 \pi}{l_{p}^{4} \Lambda}
$$

i.e.

$$
\Lambda \leq \frac{3 \pi}{l_{p}^{4} N_{U}}
$$

The problem [25] is to understand how the result in 
Equation (3) can be (re-)obtained from the point of view of a field theory where the naive estimate of the energy density, with $M_{p}=1 / l_{p}$ as ultraviolet cut-off, is given by

$$
\Lambda=\int^{M_{p}} \frac{\mathrm{d}^{3} k}{(2 \pi)^{3}} k \simeq M_{p}^{4} / 8 \pi^{2}=1 / 8 \pi^{2} l_{p}^{4} .
$$

A direct comparison between this equation and Equation (3) clearly indicates that to reproduce the bound in Equation (3) one has to introduce a limitation of the number of d.o.f. in the quantum field estimate of the energy density. Indeed, standard local field theories overcount available degrees of freedom because they fail to include the effect of gravitation [12] and the discrepancy is due to number of d.o.f. of the universe which, according to the holographic principle, has an upper bound given by the number of elementary cells on a spherical causal horizon of area, $A_{U}$, with radius equal to the inverse Hubble constant $H\left(H^{-1} \simeq 2.7 \times 10^{61} l_{p}\right)$, that is

$$
N_{U} \leq \frac{A_{U}}{l_{p}^{2}} \simeq \frac{1}{H^{2} l_{p}^{2}} \simeq 10^{122} .
$$

In the absence of a unified theory of gravity and quantum fields, in this letter a ,"crude", new method is proposed to include in the calculation of the energy density of a field theory the limitation of the number of d.o.f. in agreement with the entropy bound.

\section{Holographic Bound in Quantum Field Energy Density}

An interesting attempt in understanding the value of the cosmological constant in particle physics has been done [26] by considering the energy — not the energy densityof a quantized field in a box of size $L$. The energy is (volume * energy density) and therefore it is of order $L^{3} M_{p}^{4}$. By assuming that the lagrangian of the theory describes all state of the system excluding those for which it has already collapsed to a black-hole, a relation between the infrared cut-off, $L$, and the ultraviolet cut-off, $M_{p}$, arises in such a way that the cosmological constant turns out

$$
\Lambda \simeq\left(l_{p} L\right)^{-2}
$$

which gives (within an order of magnitude) the observed value of $\Lambda$ if one identifies $L$ with the Hubble constant ( see ref. [26] for details).

However [27], since $\Lambda \simeq 1 / L^{2}$ and in the standard cosmological model, in a dominated matter era, $L \simeq R(t)^{3 / 2}$ ( $R(t)$ is the scale factor), then $\Lambda \simeq 1 / R(t)^{3}$ which corresponds to an equation of state with $w=0 \quad(w=p / \rho$ where $p$ is the pressure and $\rho$ the energy density of the system) rather than the value $w \simeq-1$ required for the dark energy by the analysis of cosmological data [2831].

Let us rather consider the evaluation of the energy density of a quantum field by imposing the holographic entropy bound, valid for any physical system.

In general, if one covers an area $A$ by elementary cells of area $A_{p}$, the number of the d.o.f. in the considered area is $\leq A / A_{p}$.

For a generic scale related to a momentum $k$, $l_{k}=1 / k$ and $l_{k}>l_{p}$, the previous limit on the number of d.o.f of the cell of area $l_{k}^{2}$ is

$$
N_{k} \leq \frac{l_{k}^{2}}{A_{p}}
$$

therefore

$$
k \leq \frac{1}{l_{p} \sqrt{N_{k}}}
$$

Now let us assume that

$$
l_{k}^{2} N_{k} \geq l_{p}^{2} N_{U}
$$

which is a non trivial point, that will be discussed in the next section, since it is a condition among different scales and different numbers of d.o.f. in cells with very different sizes. By previous equation one has

$$
\sqrt{N_{k}} \geq k l_{p} \sqrt{N_{U}} \text {. }
$$

and by Equation (8)

$$
k \leq \frac{1}{l_{p} N_{U}^{1 / 4}}
$$

which implies that the ultraviolet cut-off to take correctly into account the holographic entropy bound is $1 /\left(l_{p} N_{U}^{1 / 4}\right)$ and not $1 / l_{p}$ as naively expected.

Therefore the energy density of a "free" field theory turns out

$$
\Lambda \simeq \int^{1 /\left(l_{p} N_{U}^{1 / 4}\right)} \frac{\mathrm{d}^{3} k}{(2 \pi)^{3}} k \simeq \frac{1}{8 \pi^{2} l_{p}^{4} N_{U}}
$$

in agreement with the entropic bound obtained by Bousso [12] and with the experimental value of the cosmological constant $\left(\Lambda=\left(2.6 \times 10^{-3}\right)^{4} \mathrm{eV}^{4}\right)$ [28-31]. The previous ultraviolet cut-off is a direct consequence of gravitation. It disappears for $l_{p} \rightarrow 0$ and, as we shall discuss later, in a consistent calculation of the energy density for interacting fields, it should be interpreted as the typical momentum scale in the loop expansion of the gravitational effective action when standard model particles couple with external graviton legs. 


\section{Scaling Behavior of Fundamental Degrees of Freedom}

Let us now analyze the meaning of the crucial assumption in Equation (9) and let us initially consider a geometrical argument by using, for semplicity, squares rather than spherical surfarces. The covering of a square of area $A=L^{2}$ with elementary squares is an old mathematical problem [32]. The minimum number $v$ of elementary squares, of area $r^{2}$, needed to cover a large square of side length $L$ is given by [33]

$$
v=\frac{L^{2}}{r^{2}}\left[1+O\left((L / r)^{-(11-\sqrt{2}) / 7} \log (L / r)\right)\right]
$$

where the positive second term in bracket is the "extra space". Therefore, in our case if one covers the entire horizon of the universe, $A_{U}$, with elementary cells of area $l_{k}^{2}$ one has

$$
v_{k} l_{k}^{2} \geq A_{U}
$$

where $v_{k}$ is the corresponding minimum number of cells to cover the horizon.

Equation (9) is equivalent to assume that at any scale $l_{k}$ the number of d.o.f. $N_{k}$ ( in the cell of area $l_{k}^{2}$ ) rearranges in such a way to be larger than or equal to to the minimum number of cell $v_{k}$ to cover the whole area, i.e.

$$
N_{k} \geq v_{k} \text {. }
$$

Indeed, by previous Equations (14) and (15), one gets

$$
N_{k} l_{k}^{2} \geq v_{k} l_{k}^{2} \geq A_{U} \geq l_{p}^{2} N_{U},
$$

which gives Equation (9).

Note that since $N_{k}$ and $N_{U}$ are integers, if $l_{k}$ is a multiple of $l_{p}$ and one imposes the exact covering of the entire area (no "extra space"), the condition $N_{k}=v_{k}$ corresponds to a self-similar rearrangement of d.o.f at different scales. From this point of view, the consistency between the holographic entropy bound for the cosmological constant and the estimate of the energy density requires a peculiar self-similar rearrangement of the elementary d.o.f at different scales that can be seen as an infrared-ultraviolet connection.

Let now discuss a different, more tradictional, approach to the meaning of the condition in Equation (11) which can be traslated, for example, in a gaussian type cut-off $\exp \left(-k^{2} l_{p}^{2} N_{U}^{1 / 2}\right)$ (of course an exponential cutoff works equally well).

This implies that there is a typical scale in the field theory effectively coupled with gravity. In the "fat graviton" theory $[34,35]$ this scale coincides with the effective size, $l_{g r}$, of the graviton and the local graviton coupling with the standard model (SM) particles is strongly modified. Infact, the dominant contributions to the gravitational effective action come from purely SM loops, with graviton external legs, which contribute only for wavelengths $>l_{g r}$. More precisely, with fat gravitons, massive SM (i.e. particle with mass $m_{S M} \gg 1 / l_{g r}$ ) and hard light SM pieces of loop contributions to the gravitational effective action may consistently [34] be suppressed while the soft light SM contribution are not, i.e. there is no robust contribution to the cosmological constant from momentum scale larger than $1 / l_{g r}$. However among the diagrams contributing to the cosmological constant there is one with no graviton external legs, corresponding to the free energy density, and the graviton size is unable to suppress this contribution. As discussed in detail in ref. [34], the cosmological term in the loop expansion of the gravitational effective action is a selfinteraction of the graviton field and, by invoking the general coordinate covariance, diagrams with soft graviton external lines are related to the diagram with no graviton. From this point of view, a contribution to the cosmological constant such as

$$
\Lambda \simeq \int \frac{\mathrm{d}^{3} k}{(2 \pi)^{3}} k \exp ^{-k^{2} l_{g r}^{2}}
$$

has to be interpreted as a short-hand for contributions from diagrams with gravitons interactions.

In this respect, the cut-off in Equation (11), although obtained by general consideration on entropy bounds, gives the typical momentum scale in the loop expansion of the gravitational effective action when SM particles couple with external graviton legs.

The meaning of the graviton size comes by combining the two previous different points of view, i.e. by imposing $l_{g r}=l_{p} N_{U}^{1 / 4}$. Indeed, by entropy bound, the number of fundamental d.o.f. in a single cell of area $l_{g r}^{2}, N_{g r}$, turns out to be

$$
N_{g r} \leq \frac{l_{g r}^{2}}{l_{p}^{2}}=N_{U}^{1 / 2} .
$$

On the other hand, the number of cell of size $l_{g r}$ one needs to cover the entire horizon is $v_{g r}=A / l_{g r}^{2}$ and it turns out

$$
v_{g r}=\frac{A}{l_{g r}^{2}} \geq \frac{l_{p}^{2} N_{U}}{l_{p}^{2} N_{U}^{1 / 2}}=N_{U}^{1 / 2}
$$

By previous Equations (18) and (19), the crucial condition in Equation (15), i.e. $N_{g r} \geq v_{g r}$, can be satisfied only by imposing $N_{g r}=v_{g r}$, that is the size of the graviton is such that the number of cells of area $l_{g r}^{2}$ one needs to cover the whole horizon is exactly equal to the number of fundamentl d.o.f. $N_{g r}$ per cell.

\section{Comments and Conclusions}

It is interesting to note that Equation (5) can be obtained from an analysis of the representations of direct product 
of $S U(2)$ groups, each group related with an elementary binary system [30] and this clarifies the connection between the results in the previous sections and elementary quantum binary degrees of freedom.

There is another crucial aspect that has to be stressed. The final results in Equations (11) and (12) do not require the saturation of the bounds in Equations (2) and (8)-(11): the cosmological constant and the number of d.o.f. turn out to have upper limits. If one applies the saturated limits, the cosmological constant would be $\Lambda \simeq\left(1 / L l_{p}\right)^{2}$ with the wrong equation of state, as previously discussed, and one could obtain inconsistent results. Rather $N_{U}$ should be considered as the maximum number of d.o.f. of a causal horizon if one uses elementary cells of size $l_{p}$, but any other elementary cell size can be used in discussing the entropy bound, and the contribution of these d.o.f. to the energy momentum tensor is $\simeq g_{\mu \nu} / l_{p}^{4} N_{U}$ corresponding to $w=-1$ in the equation of state. Equation (11) is based on holographic entropy bound and on the crucial condition in Equation (9) and its meaning is that, because of gravity, not all d.o.f. that a field theory apparently supplies can be used for consistently storing information.

Another comment concerns the result that the effective ultraviolet cut-off depends on the number of degrees of freedom due to the infrared-ultraviolet connection. An example, not directly related with the proposed approach, comes from string theories which require a non-commutative space-time [37,38], that is, in the canonical formulation,

$$
\left[x_{\mu}, x_{v}\right]=\theta_{\mu v},
$$

where $x_{\mu}$ are the space-time coordinates and $\theta_{\mu \nu}$ are fixed quantities.

In particular settings (see ref. [36-39]), one can introduce a unique parameter $\theta$ to quantify the non commutative effects. In non-commutative scalar field theory, due to the infrared-ultraviolet connection, in the evaluation of the two-point function at momentum scale $k$, the effective ultraviolet cut-off, $M_{\text {eff }}$, is given by [37-40]

$$
M_{\text {eff }}^{2}=M^{2} /\left(1+\theta^{2} M^{2} p^{2}\right)
$$

where $M$ is the ultraviolet cut-off of the commutative theory. By choosing $M=M_{p}$ and defining the number of degrees of freedom in the area $\theta$ as $N_{\theta}=\theta / l_{p}^{2}$ and in the area $l_{k}^{2}=1 / k^{2}$ as $N_{k}=l_{k}^{2} / l_{p}^{2}$, one gets

$$
M_{\text {eff }}^{2}=M_{p}^{2} /\left(1+N_{\theta}^{2} / N_{k}\right)
$$

which shows that the effective cut-off depends on the number of d.o.f. at the momentum scale $k$ and at the non-commutativity scale.

Finally, since the cosmological constant is directly related to the zero point energy density, one could comment by using statements related to Casimir effect. In- deed, in discussions of the cosmological constant, the Casimir effect is often invoked as decisive to rule out the possibility of an ultraviolet cut-off which gives result in accord with the experimental value.

However, Casimir effects can be formulated and $\mathrm{Ca}-$ simir forces can be computed without reference to zero point energies [41]. They are relativistic, quantum forces between charges and currents. The Casimir force (per unit area) between parallel plates vanishes as $\alpha$, the fine structure constant, goes to zero, and the standard result, which appears to be independent of $\alpha$, corresponds to the $\alpha \rightarrow \infty$ limit.

More generally, the physical role of the zero point energy is still an open problem and recent claims that vacuum fluctuactions of the electromagnetic field could be detected by experiments by Josephson junctions [42] have been definetely criticized in ref. [43,44].

In conclusion, if a quantum field theory has to be consistent with gravity, i.e. with an upper limit of storing information in a given area, there is an ultraviolet cut-off in the field mode of momentum $k$ given by Equation (11). The physical meaning of this cut-off in the fat graviton theory is the graviton size whereas in the approach proposed in this letter it originates by a selfsimilar behavior of the fundamental d.o.f.: the only "gravitating" modes are such that by covering the whole area of the system with the minimum number, $v_{k}$, of elementary cells of size $l_{k}=1 / k$, the number of d.o.f. per cell, $N_{k}$, has to be larger than or equal to $v_{k}$. In an effective field theory of SM particles interacting with gravitons, the cut-off $M_{p} / N_{U}^{1 / 4}$ has to be interpreted as the typical momentum scale in the loop expansion of the gravitational effective action when SM particles couple with external graviton legs. It could be quite possible to have some physical effects at a scale $\simeq 1 / l_{p} N_{U}^{1 / 4}$ and experiments on the possible modification of gravity are very close to study this range of distances (see for example [45]).

The cosmological constant problem is far from to be solved $[46,47]$ and it is deeply related with quantum gravity, however the obtained results clearly indicate a new kind of infrared-ultraviolet connection that is worth to be investigated.

\section{Acknowledgements}

The author thanks M.Consoli and D. Zappala' for useful comments and suggestions and the Theoretical Physics Department of Bielefeld University for hospitality.

\section{REFERENCES}

[1] C. F. von Weizsaker, Komplemerterritat and I. Logik, Naturwissenschaflen, Vol. 42, 1955, pp. 521-529.

[2] J. A. Wheeler, "It for Bit," In: L. Keldysh and V. Fein- 
berg, Eds., Sakharov Memorial Lectures on Physics, Vol. 2, Nova Science, New York.

[3] R. Penrose, In: C. J. Isham, R. Penrose and D. Sciama, Eds., Quantum Gravity, an Oxford Symposium, Clarendon Press, Oxford, 1975.

[4] S. Doplicher, K.Fredenhagen and J. E. Roberts, Communications in Mathematical Physics, Vol. 172, 1995, pp. 187-220. doi:10.1007/BF02104515

[5] C. Rovelli, Classical and Quantum Gravity, Vol. 28, 2011, Article ID: 153002 . arxive: 1102.360

[6] C. Rovelli, "Zakopane Lectures on Loop Gravity," arxive: 1102.360 .

[7] S. Muhki, Classical and Quantum Gravity, Vol. 28, 2011, Article ID: 153001.

[8] M. Blau and S. Theisen, General Relativity and Gravitation, Vol. 41, 2009, p. 743.

[9] J. D. Bekenstein, Physical Review D, Vol. 7, 1973, pp. 2333-2346. doi:10.1103/PhysRevD.7.2333

[10] J. D. Bekenstein, Physical Review D, Vol. 9, 1974, p. 3292.

[11] J. D. Bekenstein, Physical Review D, Vol. 23, 1981, p. 287. doi:10.1103/PhysRevD.9.3292

[12] R. Bousso, Journal of High Energy Physics, Vol. 9907, 1999, p. 4.

[13] R. Bousso, Physical Review Letters, Vol. 90, 2003, Article ID: 121302.

[14] R. Bousso, Review of Modern Physics, Vol. 74, 2002, p. 285. doi:10.1088/1126-6708/1999/07/004

[15] G. Hooft, "Dimensional Reduction in Quantum Gravity," In: In: A. Alo, J. Ellis and S. Randjbar-Daemi, Eds, Salanfestschrift, World Scientific, Singapore, 1993, p. 284.

[16] L. Susskind, Journal of Mathematical Physics, Vol. 36, 1995, p. 6377. doi:10.1063/1.531249

[17] D. A. Lowe, J. Polchinsky, L. Susskind, L. Thorlacius and J. Uglum, Physical Review D, Vol. 52, 1995, pp. 69977010. doi:10.1103/PhysRevD.52.6997

[18] E. Witten, Advances in Theoretical and Mathematical Physics, Vol. 2, 1998, p. 253.

[19] J. M. Maldacena, "Gravity, Particle Physics and Their Unification," arxive: hep-ph/0002092

[20] T. Jacobson, Physical Review Letters, Vol. 75, 1995, pp. 1260-1263. doi:10.1103/PhysRevLett.75.1260

[21] T. Padmanabhan, Classical and Quantum Gravity, Vol. 19, 2002, p. 5387.

[22] T. Padmanabhan, "Thermodynamiacl Aspect of gravity. New insights," arxive 0911.5004.

[23] E. P. Verlinde, Journal of High Energy Physics, Vol. 1104, 2011, p. 29.

[24] T. Banks, hep-th/0007146; hep-th/0306074.

[25] S. Weinberg, Physical Review Letters, Vol. 59, 1987, pp. 2607-2610. doi:10.1103/PhysRevLett.59.2607
[26] A. Cohen, D. Kaplan and A. Nelson, Physical Review Letters, Vol. 82, 1999, pp. 4971-4974. doi:10.1103/PhysRevLett.82.4971

[27] S. D. H. Hsu, Physical Letters B, Vol. 594, 2004, pp. 1316. doi:10.1016/j.physletb.2004.05.020

[28] R. A. Knop, et al., The Astrophysical Journal, Vol. 598, 2003, p. 102. doi:10.1086/378560

[29] P. Astier, et al., Astronomy \& Astrophysics, Vol. 447, 2006, p. 31. doi:10.1051/0004-6361:20054185

[30] A. Kogut, et al., The Astrophysical Journal, Vol. 665, 2007, p. 355. doi:10.1086/519754

[31] M. Tegmark, et al., Physical Review D, Vol. 74, 2006, Article ID: 123507. doi:10.1103/PhysRevD.74.123507

[32] P. Erdos and R. L.Graham, Journal of Combinatorial Theory, Series A, Vol. 19, 1975, pp. 119-123. doi:10.1016/0097-3165(75)90099-0

[33] F. Chung and R. L. Graham, Journal of Combinatorial Theory, Series A, Vol. 116, 2009, pp. 1167-1175. doi:10.1016/j.jcta.2009.02.005

[34] R. Sundrum, Physical Review D, Vol. 69, 2004, Article ID: 044014. doi:10.1103/PhysRevD.69.044014

[35] R. Sundrum, Nuclear Physics B, Vol. 690, 2004, pp. 302330. doi:10.1016/j.nuclphysb.2004.05.011

[36] T. Gornitz, International Journal of Theoretical Physics, Vol. 27, 1988, pp. 527-542. doi:10.1007/BF00668835

[37] M. Douglas and N. Nekrasov, Reviews of Modern Physics, 73, 2001, pp. 977-1029. doi:10.1103/RevModPhys.73.977

[38] R. J. Szabo, Physics Reports, Vol. 378, 2003, pp. 207-299. doi:10.1016/S0370-1573(03)00059-0

[39] S. S. Gubser and S. L. Sondhi, Nuclear Physics B, Vol. 605, 2001, pp. 395-424. doi:10.1016/S0550-3213(01)00108-0

[40] P. Castorina and D. Zappala, Physical Review D, Vol. 68, 2003, Article ID: 065008. doi:10.1103/PhysRevD.68.065008

[41] R. Jaffe, Physical Review D, Vol. 72, 2005, Article ID: 021301. doi:10.1103/PhysRevD.72.021301

[42] C. Beck and M. C. Mackey, International Journal of Modern Physics D, Vol. 17, 2008, p. 71. doi:10.1142/S0218271808011870

[43] V. Branchina, M. Di Liberto and I. Lodato, Journal of Cosmology and Astroparticle Physics, 0908:011.2009.

[44] P. Jetzer and N. Strauman, Physical Letters B, Vol. 639, 2006, p. 57. doi:10.1016/i.physletb.2006.06.020

[45] S.-Q. Yang, et al., Physical Review Letters, Vol. 108 2012, Article ID: 081111.

[46] S. M. Carroll, Living Reviews in Relativity, Vol. 3, 2001, p. 1.

[47] S. Nobbenhuis, Foundations of Physics, Vol. 36, 2006, p. 613. 